\title{
Use of Computed Tomography in Dimensional Quality Control and NDT
}

\author{
Libor Beránek ${ }^{1}$, Dora Kroisová2, Štěpánka Dvořáčkováa ${ }^{2}$ Jan Urban ${ }^{1}$, Jan Šimota ${ }^{1}$, Vladislav Andronov ${ }^{1}$, Lukáš \\ Bureš $^{1}$, Lukášs Pelikán ${ }^{1}$ \\ ${ }^{1}$ Faculty of Mechanical Engineering, Czech Technical University in Prague, Technická 4, Praha 6, 16000. Czech \\ Republic. E-mail: libor.beranek@fs.cvut.cz \\ ${ }^{2}$ Faculty of Mechanical Engineering, TU Liberec, Studentska 2, 46117 Liberec 1, Czech Republic. Email: ste- \\ panka.dvorackova@tul.cz,dora.kroisova@tul.cz
}

The article deals with the application of computed tomography in dimensional quality control. The advantage of computed tomography is that the measured part is not influenced by measuring force. It is possible to measure complex parts and assemblies, their geometry, internal structure and defects in one step. The disadvantage of CT is decreasing accuracy and resolution when measuring high density materials or large parts, caused by the use of high accelerating voltage and current. The measurement result is influenced by many factors, not only the instrument itself and the set of measuring parameters, but it also largely depends on the sample material and even its geometry. Based on the requirements for dimensional inspection, an analysis of Zeiss METROTOM 1500 computed tomography X-Ray spot size was conducted. The dependence of the spot size of the X-Ray source on the accelerating voltage and current was determined for the given instrument, as well as the relation between the voxel size and the distance of the sample from the detector. Spot size and voxel size has to be in relation, since large spot size goes against small voxel size (resolution). Using calibration artefact, the influence of the acceleration voltage on the accuracy when measuring form and size of a sphere and the distance between centres of 2 spheres was evaluated.

Keywords: computed tomography, CT applications, voxel size, spot size

\section{Introduction to Computed tomography}

Computed tomography is a technique of radiographic non-destructive testing, which is used for 3D imaging of tested objects in high resolution. For this imaging, it uses the digitalization of captured X-ray that pass through the measured object. CT reconstructs the 3D structure of the object from individual 2D images, which are obtained by capturing the X-Ray attenuation on the detector. Computed tomography allows to display the internal structure of the object without its physical disruption. The principle of the method is the interpretation of the captured image of the object on the detector. The object attenuates the dose of primary radiation that enters the test object. The attenuation is governed by physical principles, specifically the law of attenuation:

$$
\bar{D}_{p}=\bar{D}_{0} \times B \times e^{-\mu d}
$$

Where $\bar{D}_{p}$ is the energy intensity of the radiation behind the object, $\bar{D}_{0}$ is the energy intensity in front of the object, $\mathrm{B}$ is the growth factor determined experimentally, e is the Euler number, $\mu$ is the attenuation coefficient and $d$ is the thickness of the measured object. The attenuation is most affected by the attenuation coefficient $\mu$ (which depends on the radiation energy, proton number and density of the material) and the thickness of the analysed material d. The object, placed on a rotary table between the radiation source and the detector, is irradiated and its digital image is further processed. Ionizing radiation detectors, which converts X-ray photons into an electrical signal are used to make the image visible, than the image is further processed. The filtered 3D model is reconstructed as a point cloud from a series of radiographic images created in this way. A point cloud is the raw scanned data of a scanned object. Not only hardware but also software is important for CT measurements, for the reconstruction of 2D images into a 3D model, in this case Metrotom OS by Zeiss was used. On the side of CT system the quality of the reconstruction depends on how many 2D images are acquired, level of noise in the image, image averaging and detector bining, resolution and noise of each image are affected by the acceleration voltage and current levels compared to part wall thickness and material proton number and many more parameters. The scanned 3D object consists of so-called voxels (Volumetric pixels). Voxel is analogous to a planar pixel, but unlike a pixel, it has a third dimension and therefore represents a value in the spatial grid [1], [2].

In addition to the visualization of the scanned object, further processing can also take place with the aid 
of the software support. Today's most complete software solution for point cloud analysis is called VG Studio and it enables a wide range of analysis tools such as nominal-actual comparison, porosity analysis, measurement of size and geometric tolerances, wall thickness analysis, analysis of fibre orientation in composite materials or analysis of internal structure of $3 \mathrm{D}$ printed parts. Tomographic data are represented by a series of 16-bit images that represent virtual sections of a sample [3], [4]. The main limitations in the measurement are the dimensions of the object and the ability of X-rays to penetrate the object. The advantage of computed tomography is that the measured part is not loaded by measuring force similar to the tactile CMMs, so it is possible to measure very light and soft materials. However, the disadvantage of CT over CMM is the lower accuracy and precision when measuring difficult to penetrate components. The measurement result is influenced by many factors, not only by the instrument itself and the set measurement parameters, but it also largely depends on the sample itself - its material and even geometry. The limitation in terms of sample selection is given only by the design of the device itself. The weight (rotary table), dimensions (space inside the CT) and material (X-ray source power) of the sample are decisive in this respect. The best results are usually obtained with materials with a low value of the attenuation coefficient (for example polymeric materials). In the case of substances with a high attenuation coefficient (heavy metals such as Co, $\mathrm{Ni}, \mathrm{Cd}, \mathrm{Fe}$ ) on the other hand we have to expect imaging artifacts caused by accidental scattering of $\mathrm{X}$ rays.

\section{Typical CT applications in quality in- spection and research}

Computed tomography may be used to analyze differences between nominal and actual measured data by color visualization or to analyze pores and internal defects in the material of the part. Following are most common tasks for computed tomography today based on our measurements.

\subsection{Measurement of object deviations from nominal data-nominal/actual comparison}

If nominal data such as a CAD model is available, it is possible to directly compare the measured data to it. In order to be able to compare the measured data with the nominal data, the correct alignment of the part is essential. See in Fig. 1 the analysis of an identical part (at the top with the alignment to the reference features according to the documentation, at the bottom the measured data on the model are aligned using the best fit method.
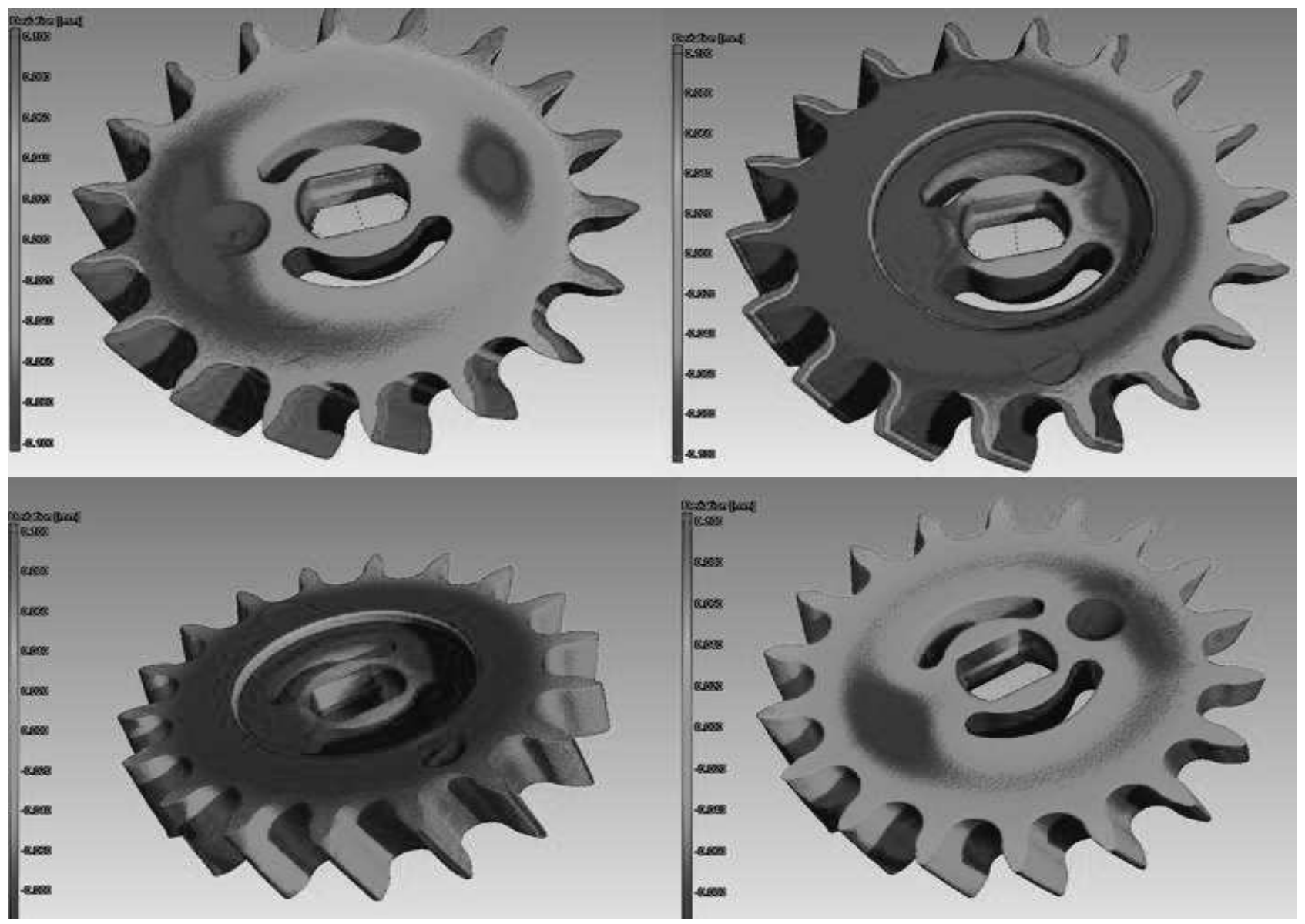

Fig. 1 Nominal/ actual comparison - influence of part alignment (alignment using datums - top, alignment using best-fit - bottom) 


\subsection{Porosity/inclusion analysis}

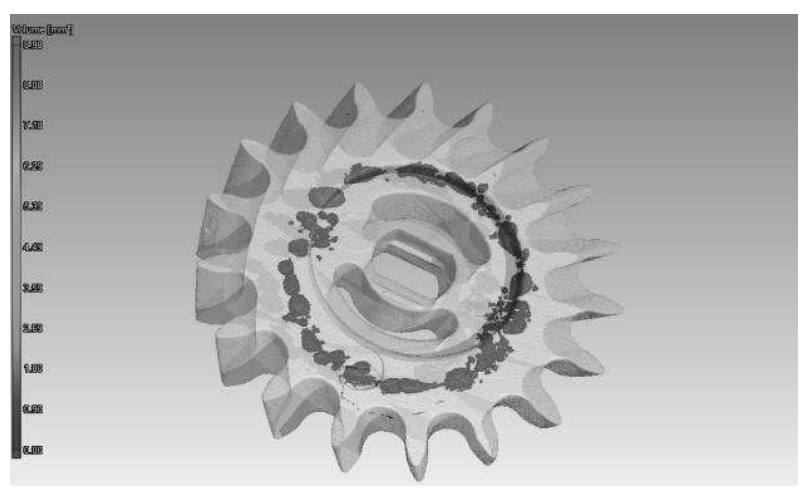

Fig. 2 Porosity analysis

Computed tomography is one of the few technologies that enables internal non-destructive analysis of materials. Thanks to this analysis, it is possible to locate various inclusions, porosity or other defects in the voxel data $[8,9]$. It is possible to obtain information on the position, size and shape of inclusions and porosity. A great advantage is the possibility of combining both analyzes, for example in the development of plastic injection molding technology. Depending on the size of the volume, defects may be highlighted in color. It is possible to add statistics of internal defects and individual annotations to individual internal defects to the output, see in Fig. 2.

\subsection{Inspection of dimensional and geometric toleran- ces}

When measuring detailed geometrical product specifications such as dimensional and form tolerances of scanned object, it is also possible to determine the individual tolerance limits and thus determine whether each specification is within tolerance limits or not. Computed tomography has a huge advantage in the field of measurement. Thanks to scanning, information can be obtained about the external as well as the internal geometric structure of the part,see in Fig. 3. Another advantage is that one scan is enough to obtain all the measured elements, this considerably shortens the inspection times for complex parts with hundreds of measured characteristics which is typical for parts that occur in the field of injection molding or high pressure die casting of aluminum alloys.

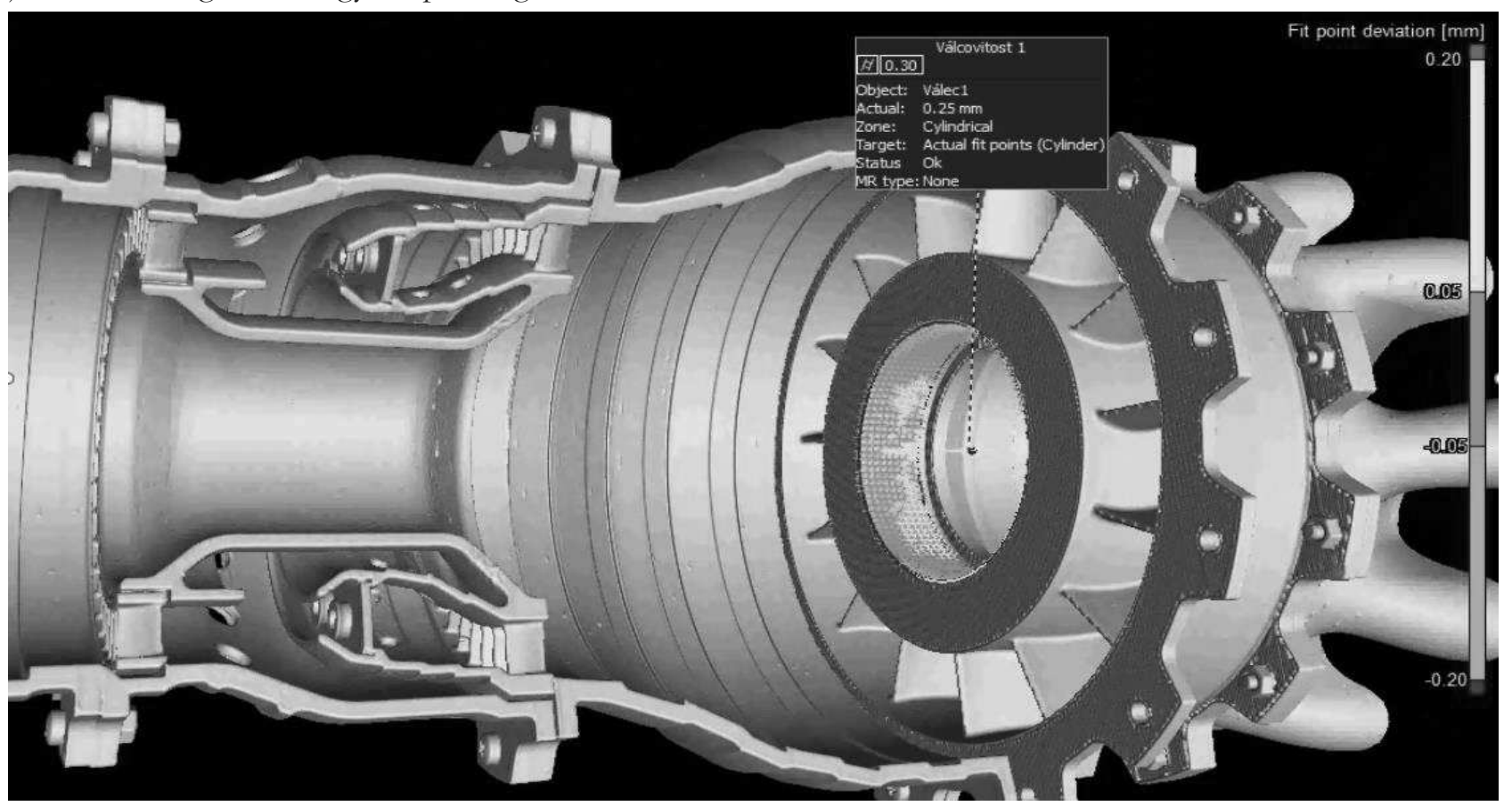

Fig. 3 Inspection of dimensional and geometric tolerances (cylindricity)

\subsection{Wall thickness analysis}

Voxel data can be used to analyze the wall thickness of scanned components. This analysis can detect places with insufficient wall thickness or conversely locate places where there is an excess of material. This knowledge can then be used to optimize the production process. Wall thickness analysis can examine objects in areas where the wall thickness is in a defined interval between the minimum and maximum value. The analysis can also check the correct location of the wall (gap). The results of the analyzed components are color-coded according to the measured distances between the walls. The analysis is effective on simple structures. This method may also be used for gap width analysis.

\subsection{Analysis and optimization of composite materials}

The use of this analysis allows the monitoring of the internal component - the reinforcement of composite material. Composite materials have been very 
popular in the last decade. The production of composite materials is a technologically and financially relatively demanding process. Fiber-reinforced composite materials are heterogeneous materials and have anisotropic properties. Computed tomography can provide a tool to evaluate the quality of a composite product, see in Fig.4. Thanks to the obtained data, it is possible to analyze the local and global orientation of the fibers, the concentration of fibers, the deviation of the fibers from the original orientation, cracks or to determine the place of delamination. Information on the behavior of the material under load can also be obtained. Computed tomography may be used to study the initiation and development of defects in a material, or to evaluate the degradation of composite materials over time. The whole sample or only few individual layers can be analyzed. The scanning of the composite material must be done with very high resolution, voxel size should be at least the same as the expected diameter of fibres or smaller.

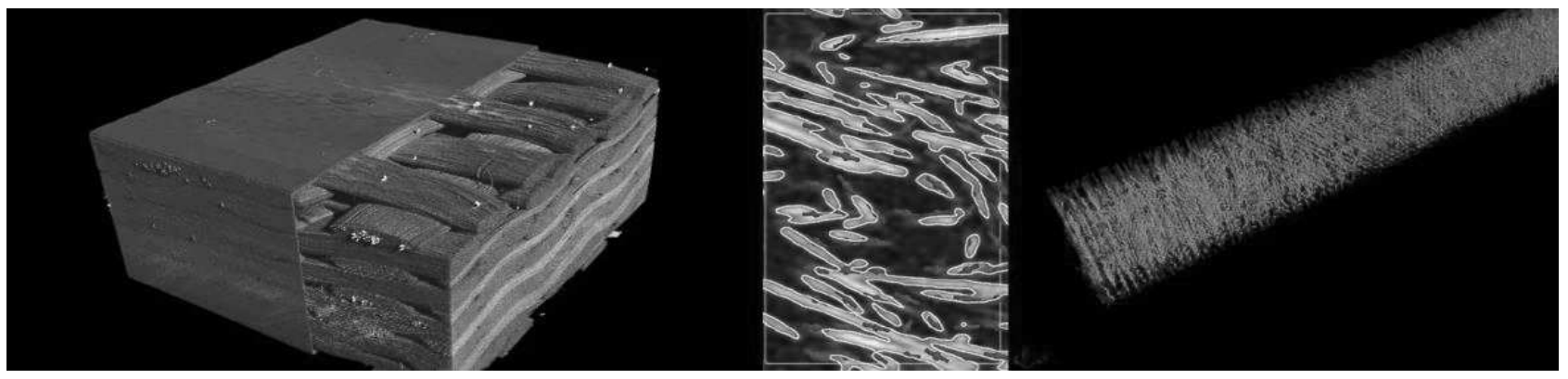

Fig. 4 Analysis of composite materials [5]

\subsection{Structure analysis of powder material}

Computed tomography is used in material analysis. This technology can be used to determine the structure of metal powder for 3D printing. Powder analysis is usually performed by laser diffraction. It is a simple and fast method that can determine the size and distribution of particles. This method takes into account the spherical shape of the powder particles, but these particles do not always have a spherical shape. By analyzing the powder using computed tomography, it is possible to monitor its quality and possibly identify undesirable aspects of the material such as sintered particles or porosity. It is also possible to determine the change in structure after recycling the powder by analysis, see in Fig.5. The use of computed tomography in the analysis of metal powder can be used to optimize the process of additive production and thus increase the resulting quality of the manufactured part [5].

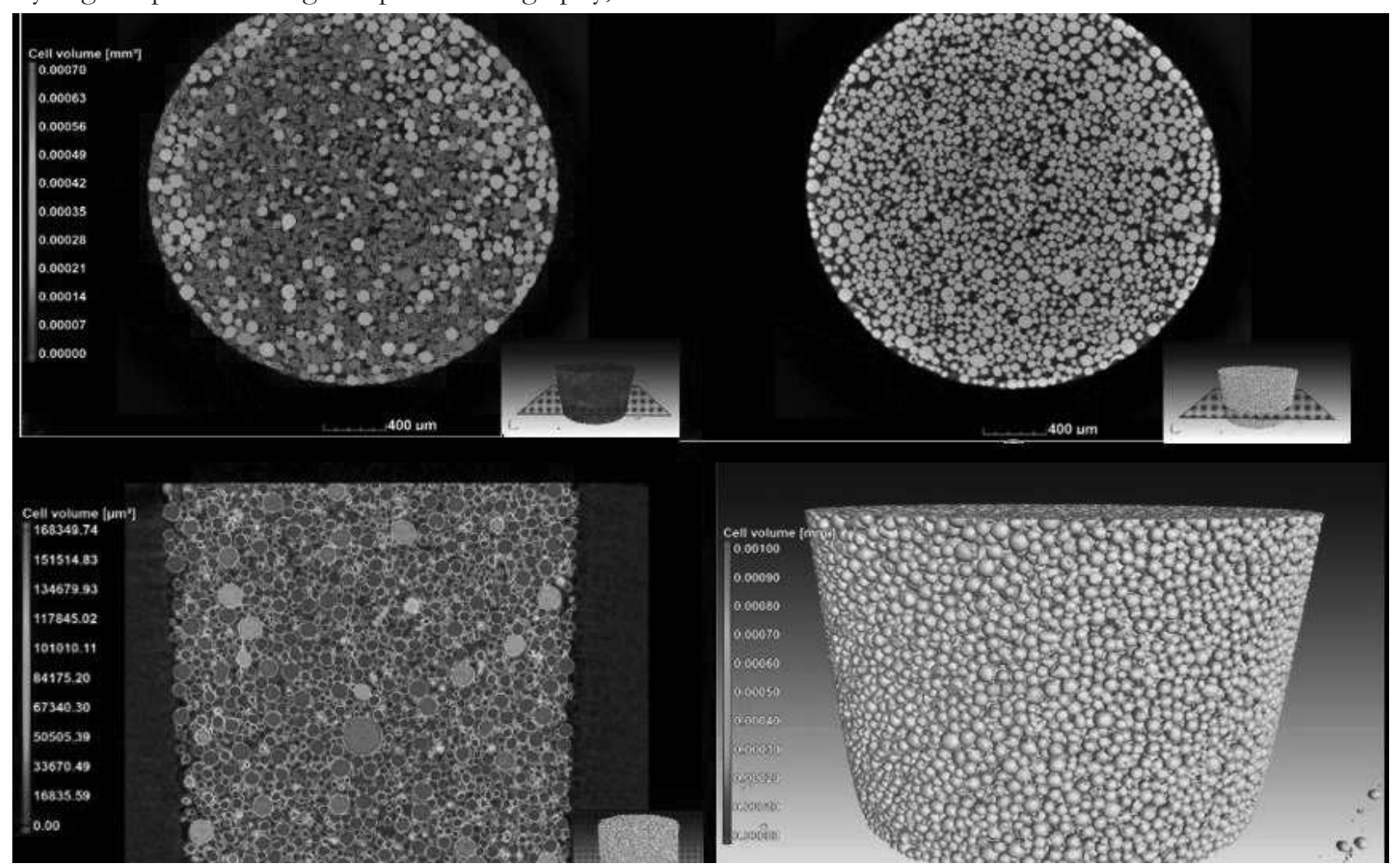

Fig. 5 Structure analysis of powder material [6] 


\subsection{Assemblies analysis}

Assemblies consisting of several parts of different materials (with different density) can also be scanned. Thanks to this analysis, it is possible to inspect the assembly kits for errors without the need for disassembly. Using computed tomography, the individual parts

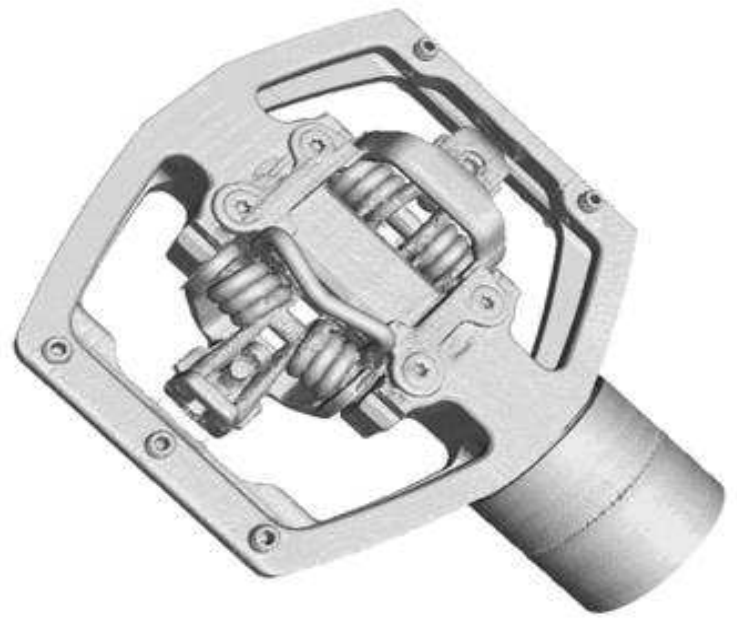

can be separated and analyzed separately. If the sample is composed of materials with different densities, different voxel intensities will be displayed in the CT data. By choosing the appropriate function for adjusting the histogram of these images, the internal structure of the material may be highlighted, see in Fig. 6.

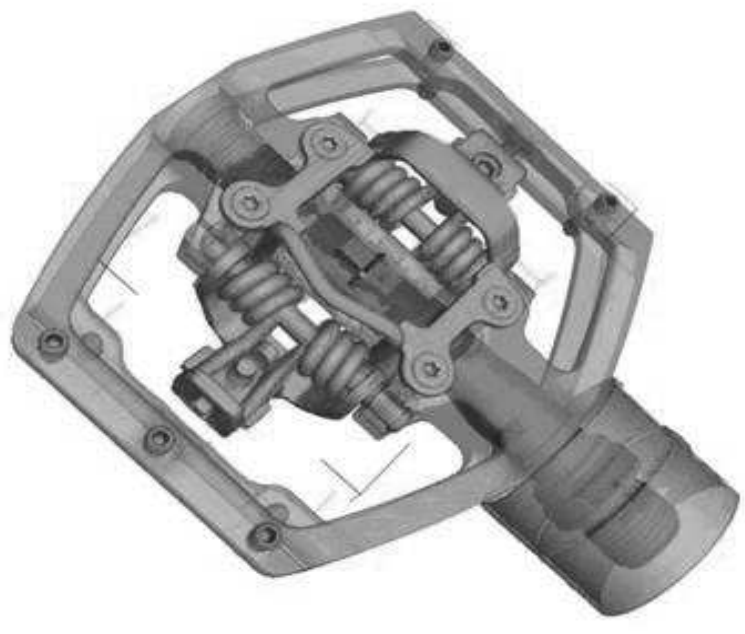

Fig. 6 Assemblies analysis (pointcloud - left, material separation - right) [7]

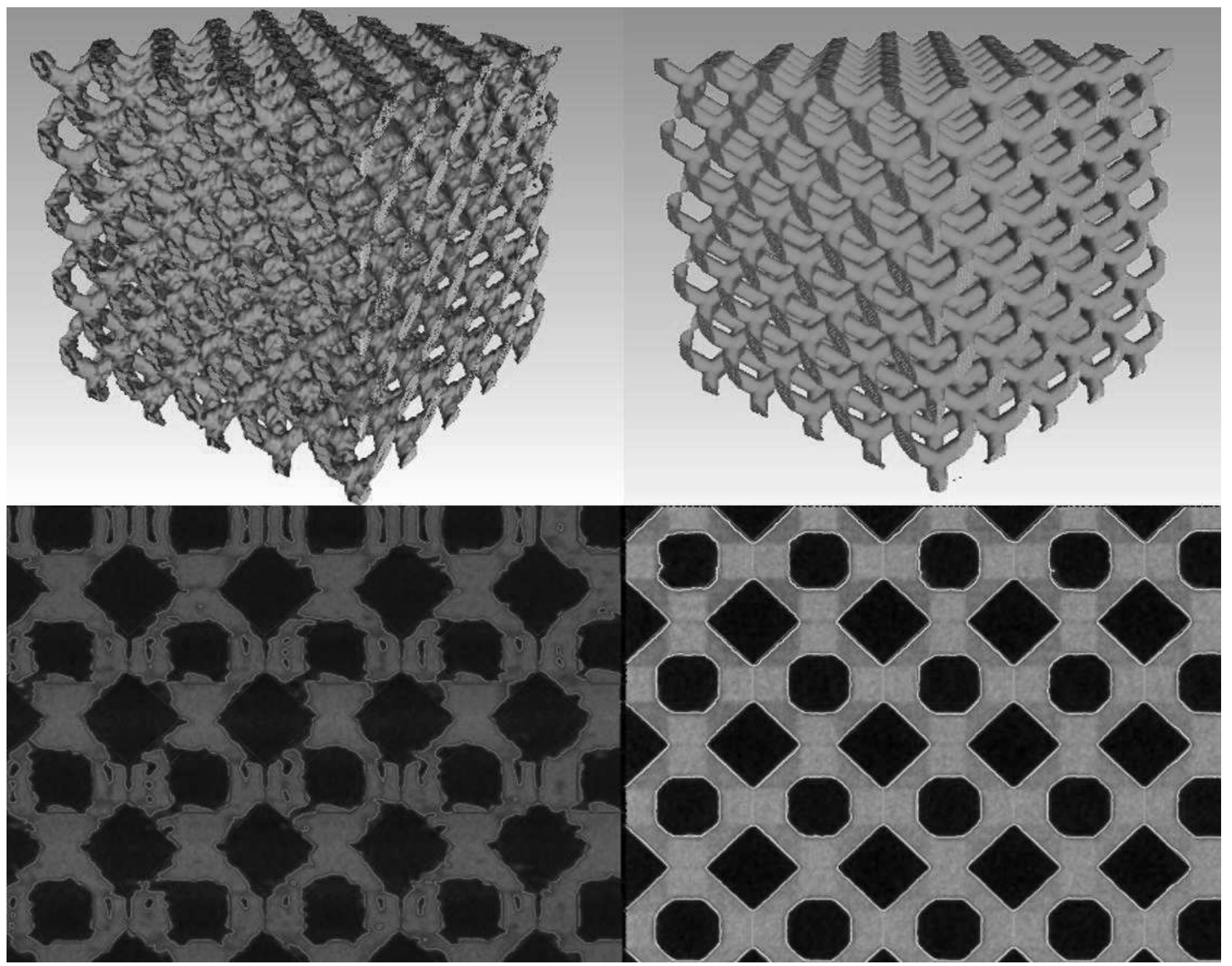

Fig. 7 Analysis of lattice structures made by 3D printing (nominal/ actual comparison for FDM and InkJet technology) 


\subsection{Analysis of lattice and lightweight structures}

Additive Manufacturing or 3D printing is a production proces that uses addition instead of material removal. This technology makes it possible to produce complex components that would not otherwise be possible with conventional technologies. Additive technologies include, for example, DMLS (direct metal laser sintering). This technology has been very popular in recent years. With DMLS, it is possible to create, for example, lightweight metal structures. However, this technology also has certain disadvantages. The printed part has different mechanical properties compared to conventional technologies. The structures are also very often porous or contain other internal defects. Using computed tomography, it is possible to obtain information about the internal and external geometric structure of complex printed components, see in Fig.7. This structure can then be analyzed to find various deformations of the shape, porosity within the part or to determine the surface characteristics. The information obtained can then be used to optimize the printing process.

It is also importatnt to stress out that $\mathrm{CT}$ is very competitive in reverse engineering applications where it is a matter of minutes or hours to get an STL file of an entire part no matter how complex it is.

\section{Analysis of X-ray spot size on energy settings for Metrotom 1500}

Metrotom 1500 is a CT equipped with X-ray tube for maximum $225 \mathrm{kV}$ acceleration voltage and 3000 $\mu \mathrm{A}$ tube current. Spot size of X-ray source affects voxel size and level of noise in each acquired image and thus for hihg resolution applications such as composite materials analysis or porosity analysis small voxel size is required. For selected CT system acceleration voltage and current were tested and 3D map of spot size in $\mu \mathrm{m}$ was constructed. It is important to emphasize that the following graphs apply only to this machine with microfocus X-ray tube.

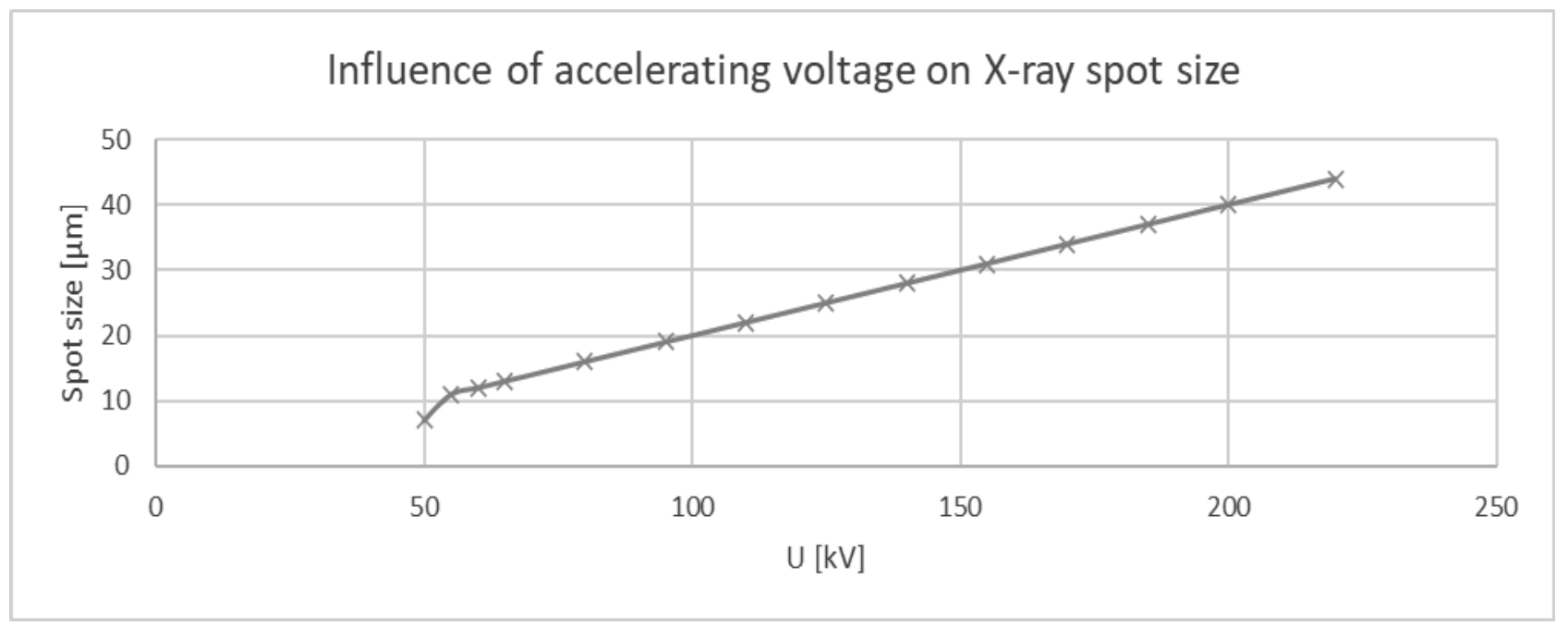

Fig. 8 Influence of accelerating voltage on X-Ray spot size

It is clear from the graph that the dependence is linear. Only when the voltage is lower than $51 \mathrm{kV}$, the size of the focus (radiation source) was reduced nonlinearly to $7 \mu \mathrm{m}$, while at lower values of the accelerating voltage the size of the focus no longer changes and remains at $7 \mu \mathrm{m}$. This is due to the technical limitations of the device, see in Fig. 8. It is advisable to keep the size of the focus spot at the lowest possible values, otherwise the object is blurred on the detector, see in Fig. 9. FOD represents Focus to Object Distance, FDD meaning Focus to Detector Distance. The ideal case is shown by the orange dashed line, the real case by the green one. There is no way to increase the focus (decrease spot size) with increasing voltage - it is a physical nature - the more energy, the greater the source of radiation. For this reason, blur is corrected by the software that processes the data, to a certain level.

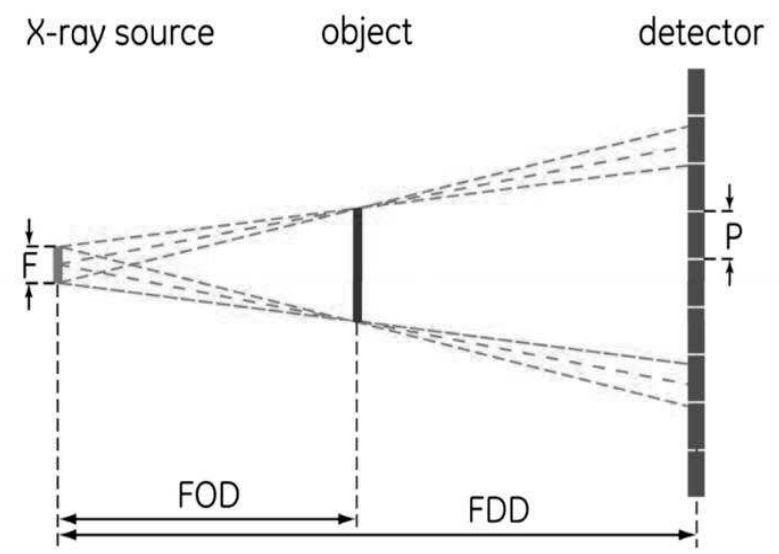

Fig. 9 Influence of spot size on detector blur [1] 


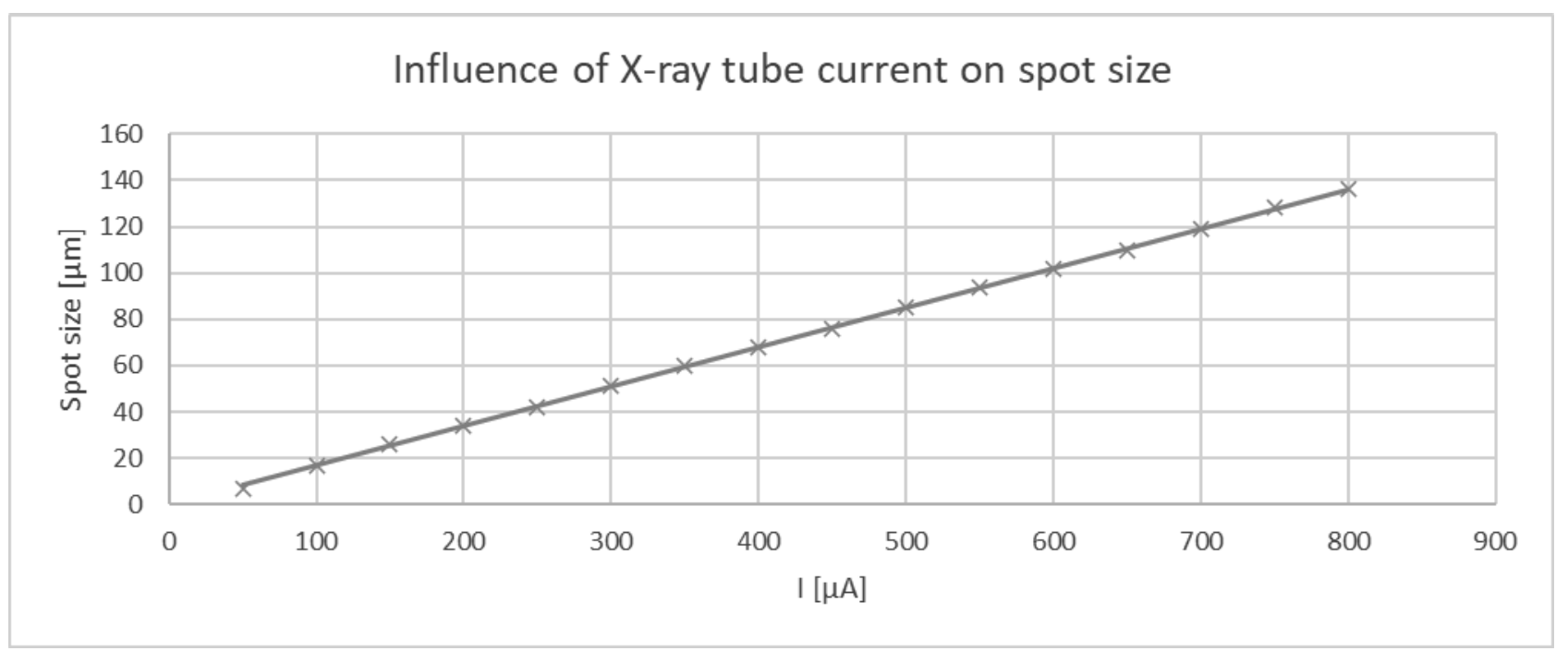

Fig. 10 Influence of $X$-Ray tube current on spot size

The dependence of the spot size on the X-Ray tube current is linear as well. The slope of the individual focus curves depends on the magnitude of the current or voltage. The maximum focus size of tested CT at maximum energy parameters reaches $500 \mu \mathrm{m}$ signaling to the user not to expect resolution better than $0.5 \mathrm{~mm}$ in high energy applications. Accelerating voltage and tube current are interacting with each other so it is appropriate to visualize spot size as a $3 \mathrm{D}$ surface of these two parameters.

\section{X-Ray spot size based on acceleration voltage and tube current}

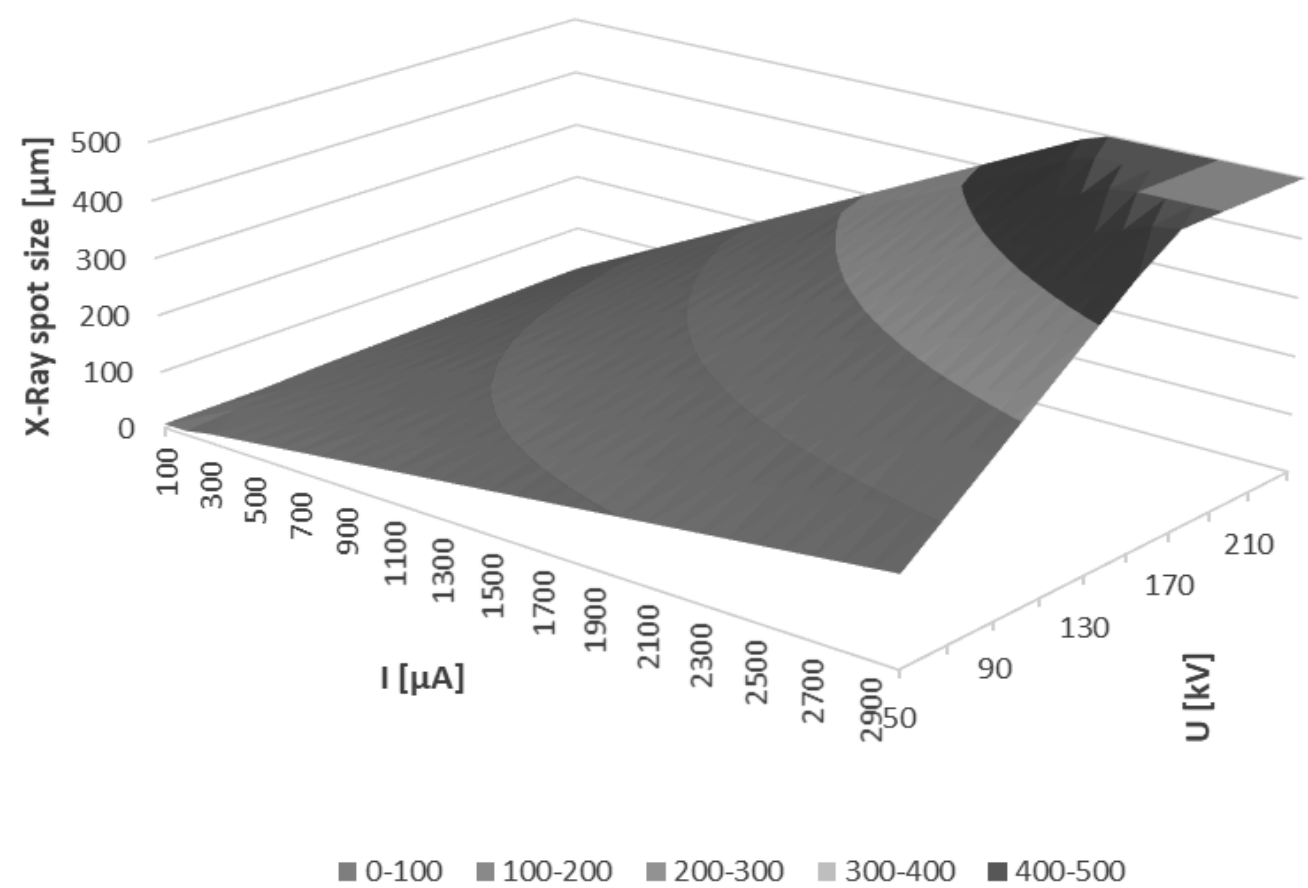

Fig. $11 X$-Ray spot size based on acceleration voltage and tube current

As mentioned earlier, the size of the spot of X-Ray source affects the achievable geometric magnification and thus the size of the voxel. The physical pixel size of the detector on the Metrotom 1500 is approximately $0.2 \mathrm{~mm}$ (the size of the detector is $400 \mathrm{x} 400 \mathrm{~mm}$ and the resolution is $2048 \times 2048$ pixels). At the highest magnification, it is possible to reach a voxel size of 5 $\mu \mathrm{m}$. 


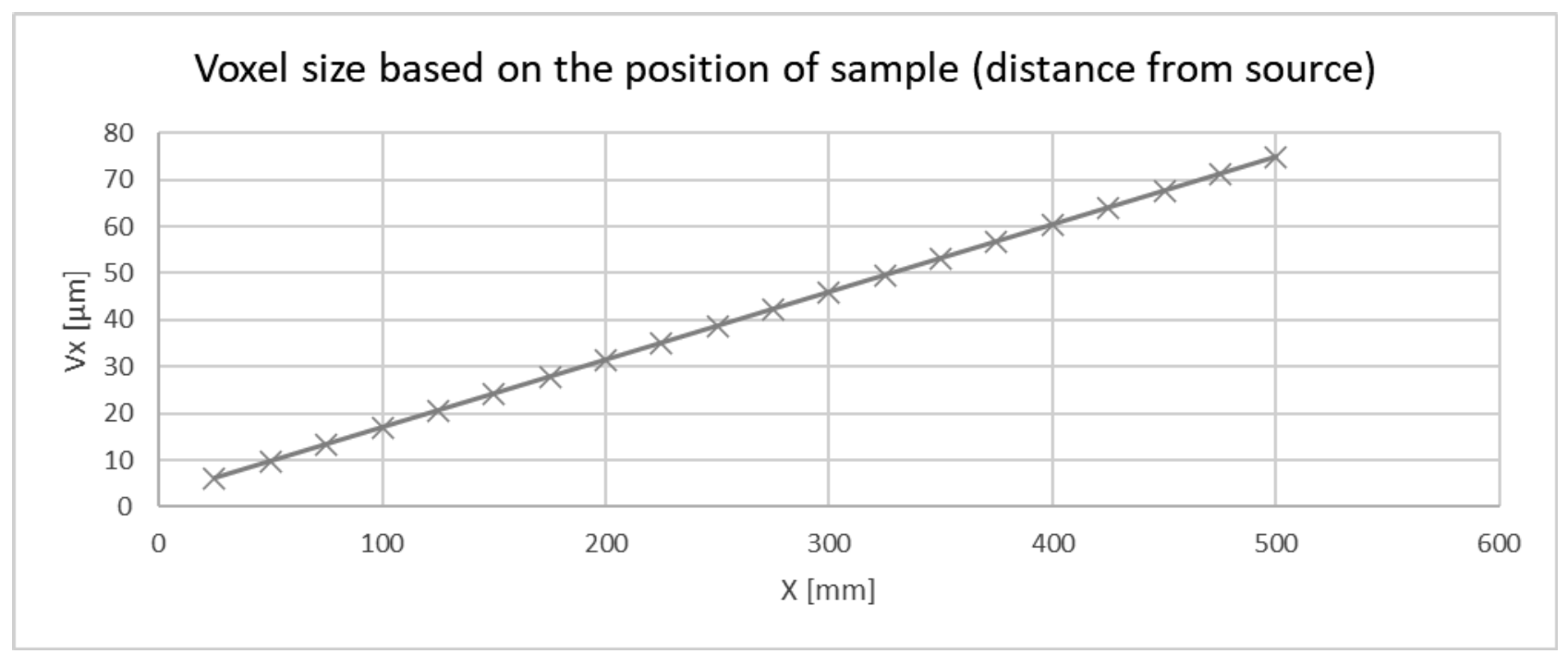

Fig. 12 Voxel size based on the position of sample in X-axis (distance from source)

4 Experimental determination of the influence of the accelerating voltage for measurement of the artefact

The influence of the accelerating voltage, as the main parameter influencing the CT measurement, was experimentally determined on the calibration artifact, which comprises of two paralel ballplates each with tungsten spheres fixed in polymer matrix, tested spheres and distances between spheres are designated in Fig. 13. The artifact was scanned using parameters, see in Tab. 1.
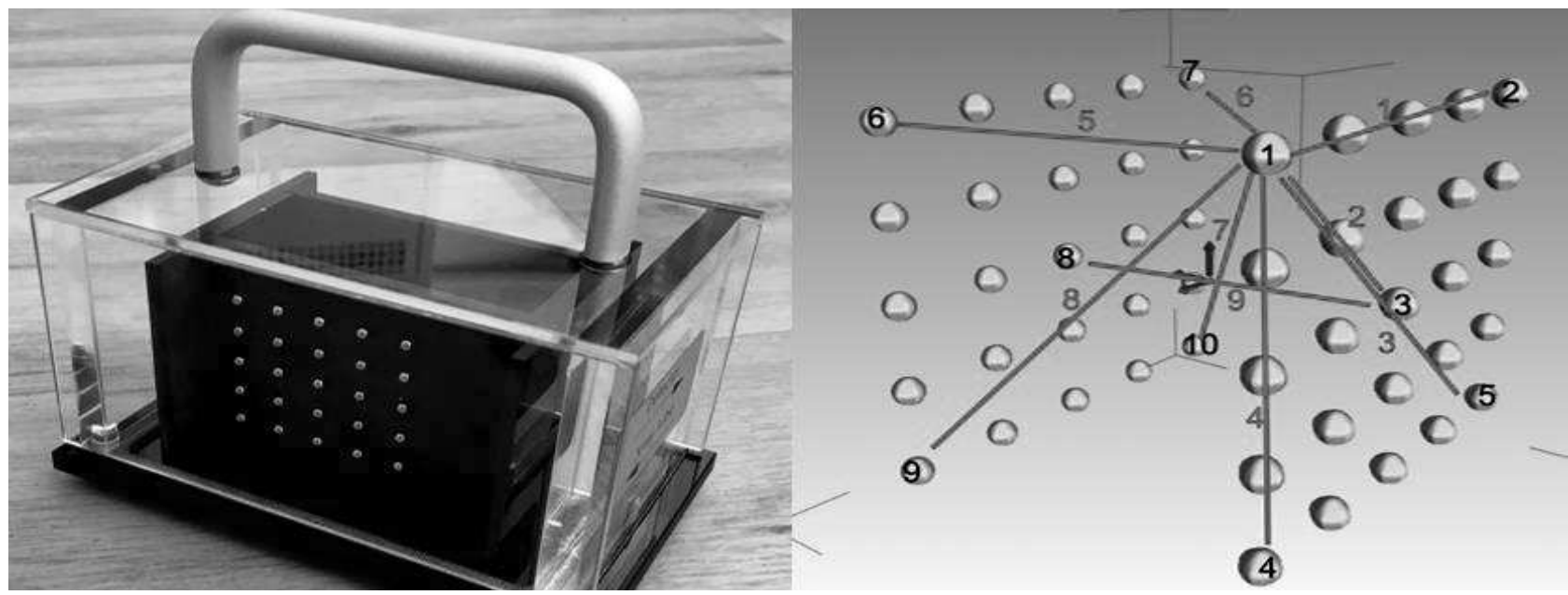

Fig. 13 Testing artefact and designation of spheres and distances

Tab. 1 Parameters of CT scan

\begin{tabular}{|c|c|c|c|c|}
\hline$U$ & $I$ & Position in X & Position in $\mathrm{Y}$ & Position in Z \\
\hline $140-180 \mathrm{kV}$ & $280 \mu \mathrm{A}$ & $300 \mathrm{~mm}$ & $0 \mathrm{~mm}$ & $180 \mathrm{~mm}$ \\
\hline
\end{tabular}

\begin{tabular}{|c|c|c|c|c|c|}
\hline $\begin{array}{c}\text { Magnifica- } \\
\text { tion }\end{array}$ & Voxel size & Spot size & Gain & $\begin{array}{c}\text { Integration } \\
\text { time }\end{array}$ & Filter \\
\hline $4,35 \times$ & $45,93 \mu \mathrm{m}$ & $39-50 \mu \mathrm{m}$ & $4 \times$ & $500 \mathrm{~ms}$ & $\mathbf{0 , 2 5} \mathbf{~ m m ~ C u}$ \\
\hline
\end{tabular}

The following graphs see Fig. 14, Fig. 15, show the measured values of the selected paramaters. The size of the sphere, the form error of the sphere and the error of distance between the spheres, which were measured from the center of the sphere to the center of the sphere. Size and form error of the sphere were calculated as an average value of 10 measurements, see Tab. 2. 


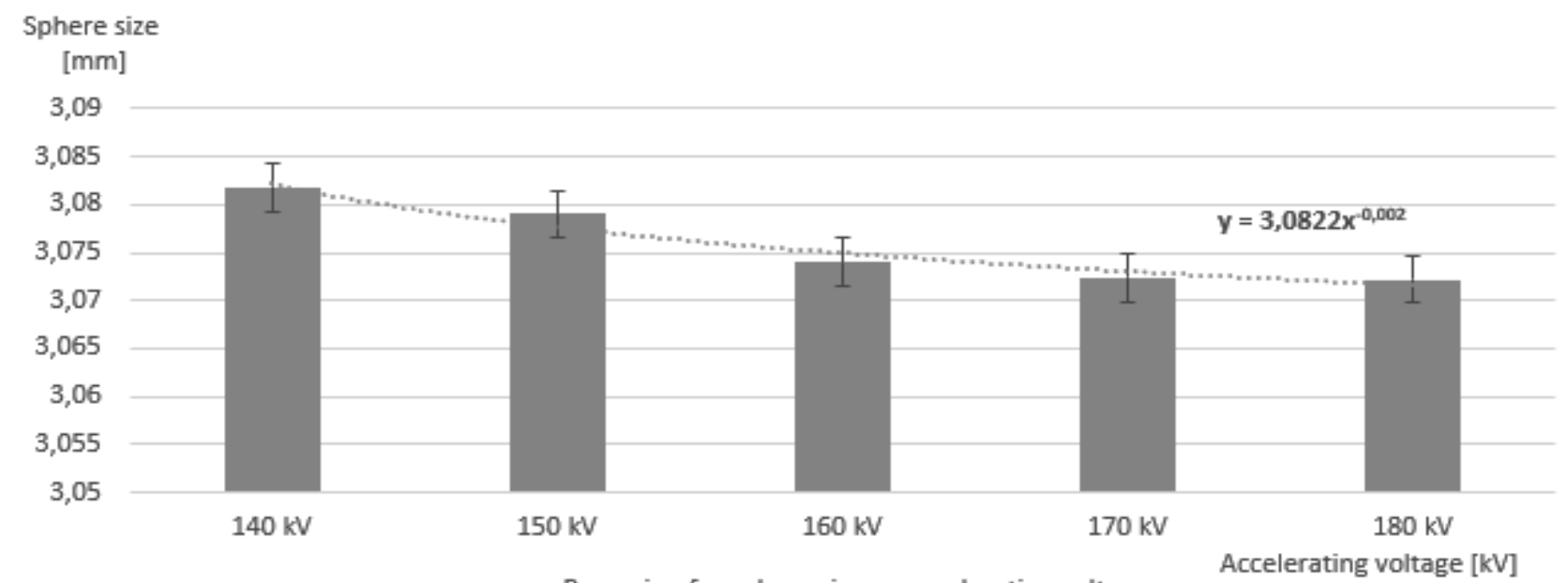

Regresion for sphere size on accelerating voltage

Fig. 14 Accelerating voltage influence on sphere size

Sphere form

error [mm]

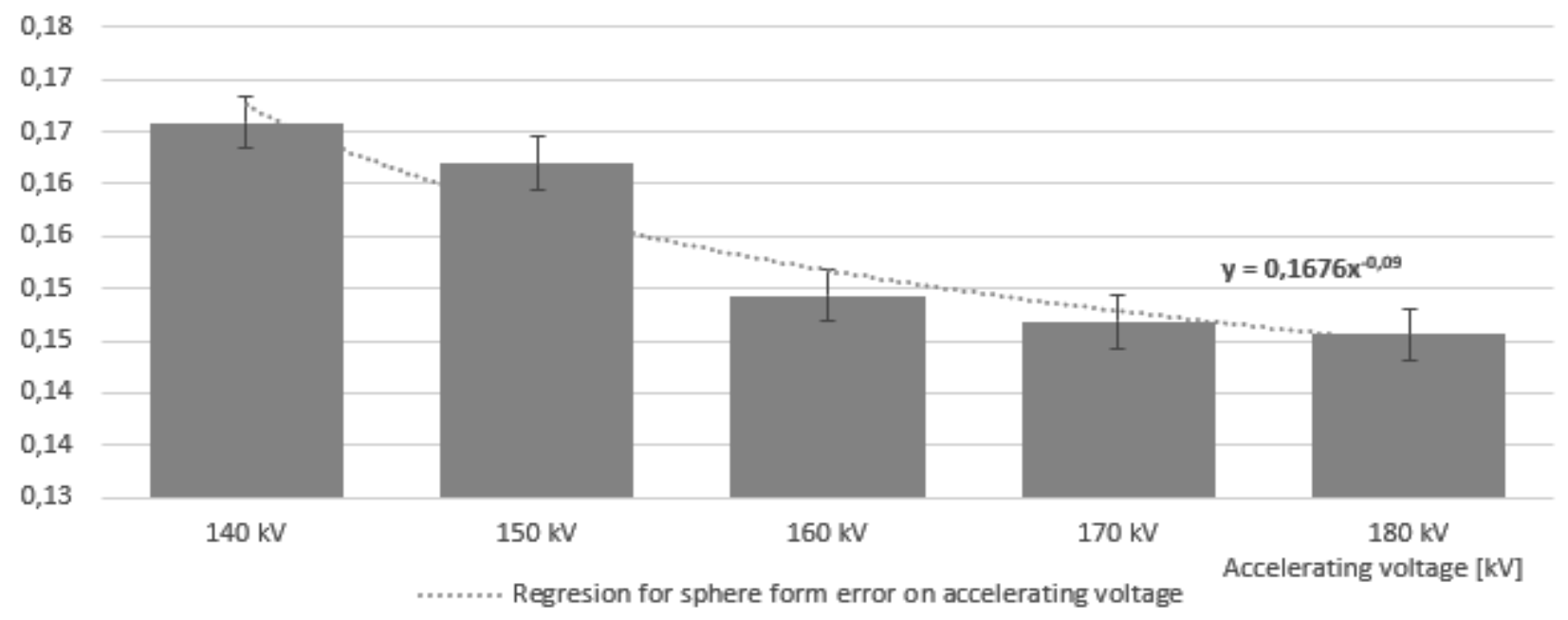

Fig. 15 Accelerating voltage influence on sphere form error

One of the most typical imaging errors can be responsible for the above-mentioned CT behavior, is called Feldkamp artifact. Feldkamp artifacts are present on scanned parts whose surfaces are horizontally oriented to penetrating X-ray. They are created because it is not possible to capture all data about the object during scanning because of radiaton scattering and this data is then missing during reconstruction. This effect causes positive or negative surface deviations in the Z-axis (rotary axis of the table). The intensity of this effect increases with increasing $\mathrm{X}$-ray angle.
This phenomenon is typical of spherical parts, where it causes form errors. This phenomenon can be eliminated on cylindrical parts by tilting the scanned part so that the smallest possible area is perpendicular to the axis of rotation ( $Z$-axis). However, elimination with spherical components is not possible by tilting, since the canopy of the sphere in the Z-axis is tangent to the direction of the $\mathrm{X}$-rays and therefore they are scattered.

Tab. 2 Measured distances between spheres and sampling standard deviation of repeated measurement

\begin{tabular}{|c|c|c|c|c|c|c|c|c|c|}
\hline $\begin{array}{c}\text { Accelerating vol- } \\
\text { tage [kV] }\end{array}$ & $\begin{array}{c}\text { Distan } \\
\text { ce 1 }\end{array}$ & $\begin{array}{c}\text { Distan } \\
\text { ce 2 }\end{array}$ & $\begin{array}{c}\text { Distan } \\
\text { ce 3 }\end{array}$ & $\begin{array}{c}\text { Distan } \\
\text { ce 4 }\end{array}$ & $\begin{array}{c}\text { Distan } \\
\text { ce 5 }\end{array}$ & $\begin{array}{c}\text { Distan } \\
\text { ce 6 }\end{array}$ & $\begin{array}{c}\text { Distan } \\
\text { ce 7 }\end{array}$ & $\begin{array}{c}\text { Distan } \\
\text { ce 8 }\end{array}$ & $\begin{array}{c}\text { Distan } \\
\text { ce 9 }\end{array}$ \\
\hline $\mathbf{1 4 0} \mathbf{~ k V}$ & 38.957 & 25.819 & 51.631 & 33.814 & 40.336 & 61.795 & 73.46 & 56.626 & 40.75 \\
\hline $\mathbf{1 5 0} \mathbf{~ k V}$ & 38.958 & 25.819 & 51.632 & 33.814 & 40.337 & 61.796 & 73.461 & 56.627 & 40.751 \\
\hline $\mathbf{1 6 0} \mathbf{~ k V}$ & 38.958 & 25.82 & 51.632 & 33.815 & 40.339 & 61.798 & 73.463 & 56.629 & 40.75 \\
\hline $\mathbf{1 7 0} \mathbf{~ k V}$ & 38.958 & 25.82 & 51.633 & 33.815 & 40.339 & 61.798 & 73.463 & 56.629 & 40.749 \\
\hline $\mathbf{1 8 0} \mathbf{~ k V}$ & 38.958 & 25.82 & 51.632 & 33.815 & 40.339 & 61.798 & 73.463 & 56.629 & 40.749 \\
\hline Std. dev. & $\mathbf{0 . 0 0 0 4}$ & $\mathbf{0 . 0 0 0 5}$ & $\mathbf{0 . 0 0 0 7}$ & $\mathbf{0 . 0 0 0 5}$ & $\mathbf{0 . 0 0 1 4}$ & $\mathbf{0 . 0 0 1 4}$ & $\mathbf{0 . 0 0 1 4}$ & $\mathbf{0 . 0 0 1 4}$ & $\mathbf{0 . 0 0 0 8}$ \\
\hline
\end{tabular}


On the other hand when measuring distance between features that are affected by Feldcamp artifact, the error is minimal as may be seen from sampling standard deviations of measured data, see in Tab. 2. The maximum std. dev. is at the level of 1.4 $\mu \mathrm{m}$ which is far below the $\mathrm{MPE}_{\mathrm{E}}$ specification for Metrotom 1500 which is $\mathrm{MPE}_{\mathrm{E}}=9+\mathrm{L} / 50 \mu \mathrm{m}$.

\section{Conclusion}

Article sumarize main application tasks of computed tomography in todays industrial quality inspection and research. Main analysis that can be conducted are nominal/actual comparison, porosity and inclusion analysis, inspection of dimensional and geometric tolerances, wall thickness analysis, fibre analysis and optimization of composite materials, structure analysis of powder material, analysis of assemblies and lattice and lightweight structures analysis. Selected tasks were demonstrated on examples.

The basic characteristics of the influence of accelerating voltage and tube current on the spot size of METROTOM 1500 was than described. It was found that the dependence of the spot size on the accelerating voltage and on the tube current are both linear within the examined scope. Spot size varies from 7 to $500 \mu \mathrm{m}$. Voxel size which is the smallest unit that can be distinguished in the point cloud has to be selected in appropriate manner to the spot size. On a small plastic parts where we can use as little energy as possible, voxel size can be on the level of $5 \mu \mathrm{m}$, on large parts or materials with high proton number where we have to use maximum energy we can't expect to find finer structures or defects than $0.5 \mathrm{~mm}$.

In the last part the influence of accelerating voltage on sphere size, sphere form error and distance between spheres was examined. In this case we have to take into consideration the Feldkamp artifact, which may be reduced by a higher accelerating voltage, but can't be eliminated completely on high density materials. On the other hand influence of Feldcamp is negligible when measuring distance between features.

\section{Acknowledgement}

This projec was supported by SGS grant of CTU in Prague and from the Center of Advanced Aerospace Technology (CZ.02.1.01/0.0/0.0/16_019/0000826), Faculty of Mechanical Engineering, Czech Technical University in Prague..

\section{References}

[1] Fundamentals of X-ray Inspection, Principles and Instrumentation. GE Imagiation at work. 2018, 2018(5), 1-53.
[2] STOCK, S. (2009). Microcomputed tomography: methodology and applications. 1. Boca Raton: CRC Press, 2009. ISBN 14-200-5876-2.

[3] HSIEH, J. (2009). Computed tomography: principles, design, artifacts, and recent advances. 2nd ed. Bellingham, Wash.: SPIE Press, 2009. ISBN 978-08194-7533-6.

[4] BOOGAARD, J., G. DIJK (1989). Non-destructive testing: proceedings of the 12th World Conference on Non-Destructive Testing, Amsterdam, The Netherlands, April 23-28, 1989. 1. New York: Elsevier, 1989. ISBN 04-448-7450-X.

[5] X-Ray Computed Tomography Scanning \& Composite Materials [online]. 2017 [cit. 202007-26]. Dostupné z: https://www.nts.com/ntsblog/x-ray-computed-tomography-scanning-composite-materials /

[6] DU PLESSIS, A., SPERLING, P., BEERLINK, A., TSHABALALA, L., HOOSAIN, S., MATHE, N., LE ROUX, S. (2018). Standard method for microCT-based additive manufacturing quality control 1: Porosity analysis. MethodsX [online]. 2018, 5, 11021110 [cit. 2020-07-26]. DOI: 10.1016/j.mex.2018.09.005. ISSN 22150161. Dostupné $\quad \mathrm{z}$ : https://linkinghub.elsevier.com/retrieve/pii/S221501611830147X

[7] GRAPHICS, Volume. VG Studio MAX internal training.

[8] BARBORA, S., NOVOTNÝ, F., PROKOP, J. (2010). Computer Tomography in Comparison with Other Testing Methods Used for the Leakage Testing of HPDC Parts. Manufacturing Technology. 2010;10(1):29-34. doi: $10.21062 /$ ujep/x.2010/a/12132489/MT/10/1/29.

[9] KOLÍNOVÁ, M., BAKALOVA, T., VOLESKÝ, L., KEJZLAR, P., KOVAČÍČ, V. (2019). Non-destructive Evaluation of Pore Size and Structural Change in Geopolymer Composite Materials with Added Fibres. Manufacturing Technology. 2019;19(1):71-76. doi: 10.21062/ujep/246.2019/a/12132489/MT/19/1/71. 